\title{
ISOMORPHISM TYPES OF TREES ${ }^{1}$
}

\section{HAIM GAIFMAN AND E. P. SPECKER}

1. Introduction. A tree is a partially ordered system $\langle A$, $\rangle$ such that for every $x \in A$ the set $P_{x}=\{y \mid y<x\}$ is well ordered by $\leqq$. The rank $\rho(x)$ of $x$ is the order type of $P_{x}$ (this is an ordinal number). The rank $\rho(T)$ of the tree is the least upper bound of $\rho(x), x \in A$. For every ordinal $\alpha, R_{\alpha}(T)$, or simply, $R_{\alpha}$, is the set of all elements of rank $\alpha$.

A subset $A^{\prime}$ of $A$ is full if for every $x \in A^{\prime}$ we have $P_{x} \subseteq A^{\prime}$; if $A^{\prime}$ is full then the rank of an element in the subtree $\left\langle A^{\prime}, \leqq\right\rangle$ is the same as its rank in $\langle A, \leqq\rangle$. A node of $T$ is a subset $N$ of $A$ of the form $\{x \mid x \in A$ and $\left.P_{x}=P_{y}\right\}$ for some $y \in A$. Clearly all elements of a node $N$ have the same rank, and this ordinal is called the rank of $N$.

A path of $T$ is a subset of $A$ which is full and linearly (totally) ordered by $\leqq$. Obviously every path is well ordered by $\leqq$, every path can be extended to a maximal path and every totally ordered subset of $A$ can be extended to a path.

A normal $\aleph_{\alpha}$ tree is a tree $\langle A, \leqq\rangle$ of rank $\omega_{\alpha+1}$ having the following properties:

(N1) $R_{\xi}$ is of power $\boldsymbol{N}_{\alpha}$ for all $\xi, 0<\xi<\omega_{\alpha+1}$.

(N2) Nodes of rank $\xi+1$ are of power $\aleph_{\alpha}$ and nodes whose rank is a limit ordinal (including 0 ) are of power 1.

(N3) If $x \in A$ and $\rho(x)<\eta<\omega_{\alpha+1}$ then there is a $y \in R$, for which $x<y$.

(N4) Every path is of power $<\boldsymbol{\aleph}_{\alpha+1}$. (Or, equivalently, every path has order type $<\omega_{\alpha+1}$.)

(N5) If $A^{\prime}$ is a path of power $\left\langle\aleph_{\alpha}\right.$ then there is an $x \in A$ such that $y \leqq x$ for all $y \in A^{\prime}$.

Normal $\boldsymbol{\aleph}_{\alpha}$ trees are of power $\boldsymbol{\aleph}_{\alpha+1}$. In the case $\boldsymbol{\aleph}_{\alpha}=\boldsymbol{\aleph}_{0}$ condition (N5) follows from (N3) and the notion of a "normal tree" coincides with Kurepa's notions of "suites distinguées" [1] and "suites $(s)$ " [2]. (In [1] nodes of limit rank are of infinite power; no proposed theorem is affected by this difference.) We add (N5) to get a nontrivial generalization to higher powers.

The existence of normal $\boldsymbol{\aleph}_{\alpha}$ trees implies that $\boldsymbol{\aleph}_{\alpha} \boldsymbol{\aleph}_{\xi}=\boldsymbol{\aleph}_{\alpha}$ for all $\xi<\alpha$. Indeed (N2), (N3), and (N5) imply that $\left|R_{\omega \xi}\right|$ (the power of $R_{\omega \xi}$ ) is $\boldsymbol{\aleph}_{\alpha}^{\aleph_{\xi}}$ while by (N1) $\left|R_{\omega \xi}\right|=\boldsymbol{\aleph}_{\alpha}$. On the other hand if for all $\xi<\alpha$ we have $\boldsymbol{N}_{\alpha}^{\aleph_{\xi}}=\boldsymbol{N}_{\alpha}$ then $\boldsymbol{\aleph}_{\alpha}$ is regular by the König-Jourdain theorem and

Received by the editors October 12, 1962.

1 Research supported in part under No. NSF Grant GP-124. 
the existence of normal $\aleph_{\alpha}$ trees follows from [4]. The generalized continuum hypothesis implies that if $\boldsymbol{\aleph}_{\alpha}$ is regular, then $\boldsymbol{\aleph}_{\alpha}^{\boldsymbol{\aleph}_{\xi}}=\boldsymbol{\aleph}_{\alpha}$ for $\xi<\alpha$, hence it implies the existence of normal $\boldsymbol{\aleph}_{\alpha}$ trees for regular $\boldsymbol{\aleph}_{\alpha}$.

Normal $\aleph_{0}$ trees, whose existence has first been proved by $N$. Aronszajn ${ }^{2}$ are closely connected with the conjecture of Souslin. This conjecture is equivalent to the statement that every normal $\boldsymbol{\aleph}_{0}$ tree, $\langle A$, $\rangle$, has $\boldsymbol{\aleph}_{1}$ pairwise incomparable elements, that is, there is a subset $A^{\prime}$ of $A$ of power $\aleph_{1}$ such that $x \nless y$ and $y \nless x$ for all $x, y \in A^{\prime}$. All known examples of normal $\boldsymbol{\aleph}_{0}$ trees have this property. Consequently, Souslin's conjecture follows from the conjecture that any two normal $\boldsymbol{\aleph}_{0}$ trees are isomorphic. The problem whether or not this is the case is Kurepa's "premier problème miraculeux" [1].

Given any two normal $\boldsymbol{\aleph}_{\alpha}$ trees, $T_{i}=\left\langle A_{i}, \leqq i\right\rangle, i=1,2$, and any $\xi<\omega_{\alpha+1}$, the trees obtained by "truncating" $T_{1}$ and $T_{2}$ at the " $\xi$ th level" are isomorphic; that is, letting $S_{\xi}\left(T_{i}\right)$ be the set of all elements of rank $<\xi$ in $T_{i}$, the trees $T_{i}(\xi)=\left\langle S_{\xi}\left(T_{i}\right)\right.$, $\left.\varliminf_{i}\right\rangle, i=1,2$, are isomorphic. Moreover if $\xi \leqq \eta<\omega_{\alpha+1}$ then any isomorphism between $T_{1}(\xi)$ and $T_{2}(\xi)$ can be extended to an isomorphism between $T_{1}(\eta)$ and $T_{2}(\eta)$. (For the denumerable case, cf. [1, p. 102].) One might be tempted by this to conjecture that any two normal $\boldsymbol{\aleph}_{\alpha}$ trees are isomorphic, nevertheless the answer, given here, to the problem is negative:

THEOREM. If $\boldsymbol{\aleph}_{\alpha}^{\aleph_{\xi}}=\boldsymbol{\aleph}_{\alpha}$, for all $\xi<\alpha$, then there are exactly $2^{\boldsymbol{\aleph}_{\alpha+1}}$ different isomorphism types of normal $\boldsymbol{\aleph}_{\alpha}$ trees.

For two given normal $\aleph_{\alpha}$ trees $T_{i}, i=1,2$, the set of isomorphisms from $T_{1}(\xi)$ onto $T_{2}(\xi)$, where $\xi$ varies over all ordinals $<\omega_{\alpha+1}$, forms a tree if the partial order relation is taken as the relation of extension. This tree satisfies (N3) and (N5). The theorem implies that there are $T_{i}, i=1,2$, for which this tree satisfies (N4).

The proof of the theorem which is presented here shows only that there are at least $\boldsymbol{\aleph}_{\alpha+2}$ different isomorphism types of normal $\boldsymbol{\aleph}_{\alpha}$ trees. However, by an additional argument (not given in this paper) the full theorem can be deduced from the weaker one without using any part of the general continuum hypothesis.

The theorem follows readily from the following:

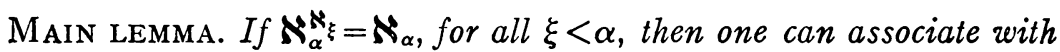
every subset $X$ of $\omega_{\alpha+1}$ of power $\boldsymbol{\aleph}_{\alpha+1}$ a normal $\boldsymbol{\aleph}_{\alpha}$ tree, $T(X)$, so that $T(X)$ and $T\left(X^{\prime}\right)$ are not isomorphic if $\left|X \cap X^{\prime}\right|<\aleph_{\alpha+1}$.

The theorem follows immediately from the main lemma and a re-

${ }^{2}$ Published in $[1$, p. 96]. A reference to this result is overlooked in [4]. 
sult of Sierpinski [3, p. 448] which states that there is a class $C$ of subsets of $\omega_{\alpha+1}$ such that $|C|=\boldsymbol{\aleph}_{\alpha+2},|X|=\boldsymbol{\aleph}_{\alpha+1}$ for all $X \in C$ and $\left|X \cap X^{\prime}\right|<\aleph_{\alpha+1}$ for all $X, X^{\prime} \in C$ which are different.

2. Sequential trees. In what follows we consider sequences of the form $\left\langle s_{0}, \cdots, s_{\lambda}, \cdots\right\rangle_{\lambda<\alpha}$, where $\alpha$ is any ordinal (including the empty sequence for which $\alpha=0)$. If $s=\left\langle s_{0}, \cdots, s_{\lambda}, \cdots\right\rangle_{\lambda<\alpha}$ then $l(s)=\alpha, l(s)$ is the length of $s$. If $t$ is a sequence and $\beta<l(t)$ then $t_{\beta}$ is the $\beta$ th member of $t$, that is, $t=\left\langle t_{0}, \cdots, t_{\beta}, \cdots\right\rangle_{\beta<l(t)}$. If $s$ is a sequence then the restriction of $s$ to $\beta, s \mid \beta$, is the sequence $t$ such that $l(t)=\operatorname{Min}(l(s), \beta)$ and $t_{\gamma}=s_{\gamma}$ for all $\gamma<l(t)$.

A sequential tree is a tree $\langle S, \leqq\rangle$ which satisfies the following conditions:

(ST1) $S$ is a set of sequences.

(ST2) If $s \in S$ then $s \mid \beta \in S$ for all $\beta \leqq l(s)$.

(ST3) $s \leqq s^{\prime}$ iff for some $\beta$ we have $s=s^{\prime} \mid \beta$.

It is easily seen that every sequential tree is a tree in which $\rho(s)$ $=l(s)$, the single element of rank 0 is the empty sequence, and nodes of limit rank are of power 1 . Conversely, if $T=\langle A, \leqq\rangle$ is a tree in which nodes of limit rank (including 0 ) are of power 1 then $T$ is isomorphic to a sequential tree. Namely, given any $x \in A$ associate with it a sequence $s$ whose length is $\rho(x)$ such that, for all $\alpha<\rho(x)$, $s_{\alpha}$ is the unique element of rank $\alpha+1$ which is $\leqq x$. In particular every normal $\aleph_{\alpha}$ tree is representable as a sequential tree.

Let $S_{\alpha}$ be the set of all sequences $s$ satisfying the following conditions:

(S1) $l(s)<\omega_{\alpha+1}$.

(S2) All the members of $s$ are ordinals $<\omega_{\alpha}$.

(S3) $\left\{\alpha \mid s_{\alpha} \neq 0\right\}$ is of power $<\boldsymbol{\aleph}_{\alpha}$.

Defining “ฏ” according to (ST3) it is immediate that $T_{\alpha}=\left\langle S_{\alpha}, \leqq\right\rangle$ is a sequential tree.

Lemma 1. If, for all $\xi<\alpha, \boldsymbol{\aleph}_{\alpha} \xi=\boldsymbol{\aleph}_{\alpha}$ then the tree $T_{\alpha}$ satisfies the conditions (N1), (N2), (N3), and (N5).

Proof. $T_{\alpha}$ satisfies (N2) and (N3) for all $\alpha$. (N5) is satisfied if $\boldsymbol{\aleph}_{\alpha}$ is regular and (N1) is satisfied if $\boldsymbol{\aleph}_{\alpha} \xi=\boldsymbol{\aleph}_{\alpha}$ for all $\xi<\alpha$.

$T_{\alpha}$ does not satisfy (N4) since the subset of $S$ consisting of all sequences $s$ for which $s_{\beta}=0$ for all $\beta<l(s)$ is a path of power $\boldsymbol{\aleph}_{\boldsymbol{\alpha}+1}$. Moreover, we have the following lemma.

Leмma 2. If, for all $\xi<\alpha, \boldsymbol{\aleph}_{\alpha} \boldsymbol{\aleph}_{\xi}=\boldsymbol{\aleph}_{\alpha}$ then every full subset of $S_{\alpha}$ of power $\boldsymbol{\aleph}_{\alpha+1}$ contains a path of power $\boldsymbol{\aleph}_{\alpha+1}$.

Proof. Let $S^{\prime} \subseteq S_{\alpha}$ be a full subset of power $\aleph_{\alpha+1}$. Let $S^{\prime}(\beta)$ be 
the subset of all sequences $s$ of $S^{\prime}$ for which $\left\{\gamma \mid s_{\gamma} \neq 0\right\}$ is of order type $<\beta$. By (S3) $S^{\prime}=S^{\prime}\left(\omega_{\alpha}\right)$. If $\beta$ is a limit ordinal then $S^{\prime}(\beta)=\bigcup_{\gamma<\beta} S^{\prime}(\gamma)$ $\left(S^{\prime}(0)=\varnothing\right)$. Thus $S^{\prime}\left(\omega_{\alpha}\right)=\bigcup_{\beta<\omega_{\alpha}} S^{\prime}(\beta)$ and consequently for some first $\xi S^{\prime}(\xi)$ is of power $\boldsymbol{\aleph}_{\alpha+1}$. $\xi$ cannot be a limit ordinal hence $\xi=(\xi-1)+1$. $\left|S^{\prime}(\xi-1)\right| \leqq \aleph_{\alpha}$, hence, for some $\eta<\omega_{\alpha+1}, l(s)<\eta$ for all $s \in S^{\prime}(\xi-1)$. On the other hand, since (N1) is satisfied (by Lemma 1), $\left\{s \mid s \in S_{\alpha}\right.$ and $\left.l(s) \leqq \eta\right\}=\bigcup_{\zeta \leq \eta} R_{\zeta}\left(T_{\alpha}\right)$ is of power $\leqq \aleph_{\alpha}$, hence $S^{\prime \prime}=\left\{s \mid s \in S^{\prime}(\xi)\right.$ and $\left.l(s)>\eta\right\}$ is of power $\aleph_{\alpha+1}$. Obviously $S^{\prime \prime} \subseteq S^{\prime}(\xi)$ $-S^{\prime}(\xi-1)$ hence $\left\{\gamma \mid s_{\gamma} \neq 0\right\}$ is of order type $\xi-1$ whenever $s \in S^{\prime \prime}$. Again by (N1) the set $\{s \mid(\eta+1)\}_{s \in S^{\prime \prime}}$ is of power $\aleph_{\alpha}$, hence there is a $t$ of length $\eta+1$ such that $S^{\prime \prime \prime}=\left\{s \mid s \in S^{\prime \prime}\right.$ and $\left.s \mid(\eta+1)=t\right\}$ is of power $\aleph_{\alpha+1}$. We claim that if $s \in S^{\prime \prime \prime}$ and $\eta \leqq \gamma<l(s)$ then $s_{\gamma}=0$. Otherwise $s_{\gamma} \neq 0$ and the order type of the indices of nonzero members of $u=s \mid \gamma$ is smaller than that of nonzero members of $s$, which is $\xi-1$, thus $\left\{\gamma \mid u_{\gamma} \neq 0\right\}$ is of order type $<\xi-1$. Since $S^{\prime}$ is full $u \in S^{\prime}$, hence $u \in S^{\prime}(\xi-1)$ but $l(u)=\gamma \geqq \eta$ contradicting the choice of $\eta$. It follows now easily that $S^{\prime \prime \prime}$ is totally ordered (its members are of length $\geqq \eta$, they coincide for ordinals $<\eta$ and are 0 from $\eta$ on). Since $S^{\prime}$ is full $\{s \mid \gamma\}_{8 \in S^{\prime \prime \prime}, \gamma<\omega \alpha+1}$ is a path of power $\aleph_{\alpha+1}$ contained in $S^{\prime}$.

3. Composition of trees. If $s$ is a sequence and $X$ is a set of ordinals then by $s \mid X$ we mean the subsequence of $s$ obtained by letting the index range over $X$ only; that is, if $X=\left\{\alpha_{0}, \cdots, \alpha_{\lambda}, \cdots\right\}$, where $\alpha_{\lambda}<\alpha_{\mu}$ for $\lambda<\mu$, then $s \mid X=\left\langle s_{\alpha_{0}}, \cdots, s_{a_{\lambda}}, \cdots\right\rangle_{\alpha_{\lambda}<l(s)},(s \mid X)_{\lambda}=s_{\alpha_{\lambda}}$. We define $s \mid \bar{X}$ to be $s \mid(l(s)-X)$ where $l(s)-X$ is the complement of $X$ relative to $l(s)$. If $s \mid X=s^{1}$ and $s \mid \bar{X}=s^{2}$ then we write $s=s^{1} *_{X} s^{2}$. (e.g., if $s^{1}=\langle 0,1\rangle, s^{2}=\langle 3,3,4\rangle$ and $X=\{0,2\}$, then $s=s^{1} *_{X} s^{2}$, where $s=\langle 0,3,1,3,4\rangle)$. It is easily seen that for every $s^{1}, s^{2}$ and $X$ there is at most one $s$ such that $s=s^{1} *_{X} s^{2}$.

If $S^{1}$ and $S^{2}$ are sets of sequences then we define $S^{1} *_{x} S^{2}$ $=\left\{s^{1} *_{X} s^{2} \mid s^{1} \in S^{1}, s^{2} \in S^{2}\right\}$. If $T^{1}=\left\langle S^{1}, \leqq\right\rangle$ and $T^{2}=\left\langle S^{2}\right.$, $\left.\leqq\right\rangle$ are sequential trees then $T^{1} *_{x} T^{2}$ is defined as the sequential tree $\left\langle S^{1} *_{x} S^{2}, \leqq\right\rangle$.

The following properties follow easily:

(C1) If $s, t \in S^{1} *_{X} S^{2}$ then $s \leqq t$ iff $s|X \leqq t| X$ and $s|\bar{X} \leqq t| \bar{X}$.

(C2) For every $\xi$ the mapping $s \rightarrow\langle s|X, s| \bar{X}\rangle$ is a one-to-one mapping of $R_{\xi}\left(T^{1} *_{X} T^{2}\right)$ onto $R_{\xi_{1}}\left(T^{1}\right) \times R_{\xi_{2}}\left(T^{2}\right)$, where $\xi_{1}$ is the order type of $\xi \cap X$ and $\xi_{2}$ is the order type of $\xi-X$.

(C3) If $T^{1}$ and $T^{2}$ are sequential trees of rank $\omega_{\beta}$ so is $T^{1} *_{X} T^{2}$.

REMARK. The isomorphism type of the compound tree depends only on the isomorphism types of the factors and on the set $X$ but not on the particular representation of the trees as sequential trees. 
Lemma 3. Let $T^{1}=\left\langle S^{1}\right.$, $\leqq$ and $T^{2}=\left\langle S^{2}\right.$, $\leqq$ be sequential trees of rank $\omega_{\alpha+1}$ and let $X$ be a subset of $\omega_{\alpha+1}$, then:

(I) If both $T^{1}$ and $T^{2}$ satisfy any of the five conditions $(\mathrm{Nj})$, $j=1, \cdots, 5$, then $U=T^{1} *_{X} T^{2}$ satisfies the same condition.

(II) If $|X|=\aleph_{\alpha+1}$ and $T^{1}$ satisfies (N4) so does $U$.

Proof of (I). For every $\xi<\omega_{\alpha+1}$ let $\xi_{1}$ be the order type of $\xi \cap X$ and $\xi_{2}$ the order type of $\xi-X$.

(N1) If $\xi>0$ then either $\xi_{1}>0$ or $\xi_{2}>0$, hence if $T^{1}$ and $T^{2}$ satisfy (N1) it follows from (C2) that $U$ satisfies it as well. (Note that in every sequential tree $R_{0}$ has one member, namely, the empty sequence.)

(N2) A node of $U$ of rank $\xi+1$ is of the form $A=\left\{s \mid s \in S^{1} *_{X} S^{2}\right.$, $l(s)=\xi+1$ and $s \mid \xi=t\}$, where $t$ is some fixed sequence of $S^{1} *_{X} S^{2}$ whose length is $\xi$. Putting $t^{1}=t \mid X$ and $t^{2}=t \mid \bar{X}$ it follows that $t^{1} \in S^{1}$, $t^{2} \in S^{2}$ and that $A^{1}=\left\{s \mid s \in S^{1}, l(s)=\xi_{1}+1\right.$ and $\left.s \mid \xi_{1}=t^{1}\right\}$ and $A^{2}=\left\{s \mid s \in S^{2}, l(s)=\xi_{2}+1\right.$ and $\left.s \mid \xi_{2}=t^{2}\right\}$ are nodes of $T^{1}$ and $T^{2}$ of ranks $\xi_{1}+1$ and $\xi_{2}+1$, respectively. If $\xi \in X$ then $A=\left\{s^{1}{ }^{*} t^{2} \mid s^{1} \in A\right\}$ and if $\xi \notin X$ then $A=\left\{t^{1} *_{X} s^{2} \mid s^{2} \in A^{2}\right\}$. It follows that either $|A|$ $=\left|A^{1}\right|$ or $|A|=\left|A^{2}\right|$. Thus if (N2) holds for both $T^{1}$ and $T^{2}$ we have $|A|=\boldsymbol{\aleph}_{\alpha}$; nodes of limit rank have power 1 since $U$ is a sequential tree, hence $U$ satisfies (N2).

(N3) If $s \in R_{\xi}(U)$ and $\xi \leqq \eta<\omega_{\alpha+1}$ then $s^{1}=s \mid X \in R_{\xi_{1}}\left(T^{1}\right)$ and $s^{2}=s \mid \bar{X} \in R_{\xi_{2}}\left(T^{2}\right)$. If both $T^{1}$ and $T^{2}$ satisfy (N3) then there are $t^{1} \in R_{\eta_{1}}\left(T^{1}\right)$ and $t^{2} \in R_{\eta_{2}}\left(T^{2}\right)$ such that $s^{1} \leqq t^{1}$ and $s^{2} \leqq t^{2}$. From (C2) and (C1) it follows that $t=t^{1} *_{X} t^{2} \in R_{\eta}(U)$ and $s \leqq t$.

(N4) If both $T^{1}$ and $T^{2}$ satisfy (N4) so does $U$. This follows from (II) of the present lemma, and the fact that either $X$ or $\omega_{\alpha+1}-X$ is of power $\aleph_{\alpha+1}$ and $T^{1} *_{X} T^{2}=T^{2} *_{\left(\omega_{\alpha+1}-X\right)} T^{1}$.

(N5) Let $A$ be a totally ordered set in $U$ of power $<\aleph_{\alpha}$. Let $\xi$ be the least upper bound of the ranks of the members of $A$. Then $A^{1}=\{s \mid X\}_{s \in A}$ and $A^{2}=\{s \mid \bar{X}\}_{s \in A}$ are totally ordered sets in $T^{1}$ and $T^{2}$, respectively, and $\xi_{1}, \xi_{2}$ are the respective least upper bounds of the ranks of the members of $A^{1}$ and $A^{2}$. If (N5) holds for both $T^{1}$ and $T^{2}$ then there are $t^{1} \in S^{1}$ and $t^{2} \in S^{2}$ such that $t^{1} \geqq s$ for all $s \in A^{1}$ and $t^{2} \geqq s$ for all $s \in A^{2}$. Moreover, as is easily seen, $t^{1}$ and $t^{2}$ can be chosen to be of ranks $\xi_{1}$ and $\xi_{2}$, respectively. From (C2) and (C1) it follows that $t=t^{1} *_{X} t^{2} \in R_{\xi}(U)$ and $t \geqq s$ for all $s \in A$.

Proof of II. Let $A$ be a path of $U$ of order type $\xi .\{s \mid X\}_{s \in A}$ is a path of $T^{1}$ of order type $\xi_{1}$. If $\xi \geqq \omega_{\alpha+1}$ and $|X|=\aleph_{\alpha+1}$ then $\xi_{1} \geqq \omega_{\alpha+1}$. Thus if every path of $T_{1}$ is of order type $<\omega_{\alpha+1}$ the same holds for $U$.

4. A class of normal trees. The following assumptions are made 
throughout this section: $\alpha$ is such that, for all $\xi$ smaller than $\alpha$, $\aleph_{\alpha} \aleph_{\xi}=\aleph_{\alpha} . T_{\alpha}=\left\langle S_{\alpha}\right.$, $\rangle$ is the sequential tree defined by (S1)-(S3) of the previous section. $T$ is some fixed normal $\aleph_{\alpha}$ tree. For every $Y \subseteq \omega_{\alpha+1}$ we define $T(Y)$ to be $T *_{Y} T_{\alpha} . X$ is a subset of $\omega_{\alpha+1}$ of power $\aleph_{\alpha+1}$ and $T(X)=\langle A, \leqq\rangle$.

LemMA 4. $T(X)$ is a normal $\aleph_{\alpha}$ tree.

Proof. By Lemma $1 T_{\alpha}$ satisfies (N1), (N2), (N3) and (N5), hence by Lemma 3 (I) these hold also for $T(X)$. Since (N4) holds for $T$ and $|X|=\boldsymbol{\aleph}_{\alpha+1}$ (N4) holds for $T(X)$ by Lemma 3 (II).

Our aim is to show that as $X$ varies over subsets of $\omega_{\alpha+1}$ the trees $T(X)$ satisfy the properties mentioned in the main lemma of the introduction. This is done in the following two lemmas.

Lemma 5. There is a full subset $B$ in $T(X)$, of power $\aleph_{\alpha+1}$, such that in the subtree $\langle B, \leqq\rangle$ every node $N$ is of power 1 whenever the rank of $N, \rho(N)$, is $\xi+1$ and $\xi \notin X$.

Proof. Put $B=\left\{s \mid s \in A\right.$ and $s_{\lambda}=0$ whenever $\left.\lambda \in X\right\}$. It is easily seen that $B$ is a full set of power $\aleph_{\alpha+1}\left(B=A^{\prime} *_{X} O_{\alpha}\right.$, where $T=\left\langle A^{\prime}, \leqq\right\rangle$ and $O_{\alpha}$ is the path of $T$ consisting of all sequences of length $<\omega_{\alpha+1}$ which are always 0 . If $s$ and $t$ are members of $B$ of length $\xi+1$ such that $s|\xi=t| \xi$, then, if $\xi \notin X$ we must have $s_{\xi}=t_{\xi}=0$ and consequently $s=t$. Thus every node of rank $\xi+1$, where $\xi \notin X$ is of power 1 .

(Since every full subset forms a sequential tree the same is trivially true for nodes of limit rank.)

LEMma 6. Let $X^{\prime} \subseteq \omega_{\alpha+1}$, and assume that there is a full subset, $B$, of $A$, of power $\aleph_{\alpha+1}$, such that every node $N$ in $\langle B$, $\leqq$ for which $\rho(N)$ $=\xi+1$ and $\xi \notin X^{\prime}$, is of power 1. Then $\left|X \cap X^{\prime}\right|=\boldsymbol{\aleph}_{\alpha+1}$.

Proof. By contradiction. Assume that $\left|X \cap X^{\prime}\right|<\boldsymbol{\aleph}_{\alpha+1}$. There is an ordinal $\eta<\omega_{\alpha+1}$ such that $X \cap X^{\prime} \subseteq \eta$. Since $R_{\eta}(T(X))$ is of power $\aleph_{\alpha}$ there is a $t \in R_{\eta}(T(X))$ such that the set $\{s \mid s \in B$, and $t \leqq s$ or $s \leqq t\}$ is of power $\aleph_{\alpha+1}$. This set is also full and it determines a subtree in which every node $N$ such that $\rho(N)=\xi+1$ where $\xi \notin X^{\prime}-X$ is of power 1 . (This is so since every node of rank $\leqq \eta$ is of power 1 , while for $\lambda>\eta$ if $\lambda \notin X^{\prime}-X$ then $\lambda \notin X^{\prime}$.) Therefore without loss of generality we can assume that $X \cap X^{\prime}=\varnothing$.

If $s$ and $t$ are two incomparable elements of $B$ (i.e., $s$ then there is a first $\xi$ such that $\xi<l(s), l(t)$ and $s_{\xi} \neq t_{\xi}$. The sequences $s \mid(\xi+1)$ and $t \mid(\xi+1)$ are two different sequences belonging to a single node of $\langle B, \leqq\rangle$ of rank $\xi+1$, hence $\xi \in X^{\prime}$ and consequently 
$\xi \notin X$. This shows that whenever $s$ and $t$ are incomparable elements of $B$ then $s \mid \bar{X}$ and $t \mid \bar{X}$ are incomparable elements of $T$. Now consider $B^{\prime}=\{s \mid \bar{X}\}_{s \in B} . B^{\prime}$ is a full subset of $S_{\alpha}$. If $\left|B^{\prime}\right|<\aleph_{\alpha+1}$ then for some $t \in B^{\prime}$ there is a subset $D$ of $B$ such that $|D|=\aleph_{\alpha+1}$ and $s \mid \bar{X}=t$ for all $s \in D$. It follows that $D$ cannot contain incomparable elements. Hence, it is totally ordered, contradicting the property (N4) which holds for $T(X)$. If $\left|B^{\prime}\right|=\boldsymbol{N}_{\alpha+1}$ then by Lemma 2 it contains a path $D^{\prime}$, of power $\boldsymbol{\aleph}_{\alpha+1}$. The set $D$ of all sequences $s$ for which $s \mid \bar{X} \in D^{\prime}$ is again of power $\boldsymbol{\aleph}_{\alpha+1}$ and has no incomparable elements, which yields the same contradiction.

From Lemmas 5 and 6 it follows immediately that if $Y, Y^{\prime} \subseteq \omega_{\alpha+1}$, $|Y|=\left|Y^{\prime}\right|=\boldsymbol{\aleph}_{\alpha+1}$ and $\left|Y \cap Y^{\prime}\right|<\boldsymbol{\aleph}_{\alpha+1}$ then $T(Y)$ and $T\left(Y^{\prime}\right)$ are not isomorphic. Thus our main lemma is proved, and this, as indicated in the introduction, implies the required result.

\section{REFERENCES}

1. G. Kurepa, Ensembles ordonnés et ramifiés, Publ. Math. Univ. Belgrade 4 (1935), $1-138$.

2. - A propos d'une généralisation de la notion d'ensembles bien ordonnés, Acta Math. 75 (1943), 139-150.

3. W. Sierpinski, Cardinal and ordinal numbers, Polska Akademia Nauk, Panstwowe Wydawnictwa Naukowe, Warsaw, 1958.

4. E. Specker, Sur un probleme de Sikorski, Colloq. Math. 2(1949), 9-12.

Columbia University,

CoRnell University and

EIdgenössische TeChNische Hochschule 